\title{
HIPOTIROIDISMO EN EL EMBARAZO
}

\section{Hypothyroidism in pregnancy}

\author{
Carla Lorena Macchia, M.D. *, Javier Augusto Sánchez-Flórez, M.D.**
}

Recibido: julio 26/07 - Revisado: noviembre 28/07 - Aceptado: noviembre 30/07

\section{RESUIMEN}

Introducción: el hipotiroidismo se presenta entre el 0,5 y 2,5\% de todos los embarazos. La frecuencia es mayor si se consideran poblaciones de riesgo, como pacientes portadoras de enfermedades autoinmunes. Durante la gestación temprana existe dependencia de hormonas tiroideas maternas, puesto que la tiroides fetal es incapaz de elaborar sus productos hasta después del primer trimestre. En este periodo, toda situación de carencia puede ejercer efectos indeseables.

Objetivo: el objetivo del presente trabajo es revisar algunos aspectos de la fisiología de la tiroides y del hipotiroidismo durante la gestación, así como su evaluación, manejo y pronóstico.

Materiales y métodos: se efectuó una búsqueda bibliográfica en bases de datos electrónicas (Medline, Cochrane) y libros de texto impresos, seleccionando los artículos considerados por nosotros de mayor relevancia científica y epidemiológica. Se consultó material publicado entre 1990 y 2007.

Discusión: la embarazada presenta cambios en la fisiología tiroidea. Ocurre un aumento del tamaño glandular y se incrementa la globulina transportadora de hormonas (TBG), en respuesta a la elevación de los estrógenos. Algunas pacientes llegan a desarrollar en el primer trimestre un hipertiroidismo transitorio, por el aumento de la gonadotrofina co-

* Ginecoobstetra. Especialista en Endocrinología Ginecológica y Reproductiva.Docente de Ginecología de la Universidad del Magdalena. Correspondencia: calle 27 carrera 2. Conjunto Residencial Quintas del Prado. Casa 11. Santa Marta, Colombia. Correo electrónico: ramasaki@hotmail.com

* Ginecoobstetra. Especialista en Endocrinología Ginecológica y Reproductiva Saludcoop IPS. Santa Marta, Colombia. riónica (hCG). Una serie de eventos ocurren como consecuencia de la interacción del eje materno con la unidad feto-placentaria, sin embargo, la embarazada normal se mantiene eutiroidea.

Durante el embarazo se debe evaluar la función tiroidea con determinación de tirotrofina (TSH) y tiroxina libre (T4L), siendo de utilidad en ciertos casos la detección de anticuerpos antitiroideos. En la terapia sustitutiva se deben utilizar dosis plenas intentándose mantener, desde el momento del diagnóstico, una T4L en el límite superior normal. Pacientes hipotiroideas previas suelen requerir un incremento de la dosis entre el 25 y el 50\%.

Las pacientes con anticuerpo antiperoxidasa (ATPO) positivo en el primer trimestre, presentan mayor riesgo de enfermedad tiroidea posparto y sintomatología psiquiátrica. En ellas deberá efectuarse seguimiento durante el periodo puerperal.

Palabras clave: hipotiroidismo, tiroides, embarazo.

\section{SUIMMARY}

Introduction: hypothyroidism is present in 0.5$2.5 \%$ of all pregnancies. Frequency becomes greater when considering risk populations (i.e. patients who are carriers of other autoimmune diseases). A foetus depends on maternal thyroid products during early gestational stages because its thyroid gland is not able to release its products until the end of the first trimester. Impaired thyroid function or iodine deficiency could thus exert undesirable effects on the foetus during the embryogenic period.

Objective: the present work is aimed at reviewing some aspects of the physiology of the thyroids and 
hypothyroidism during pregnancy, how to evaluate it, its management and prognosis.

Materials and methods: a bibliographic search was made of electronic databases (Medline, Cochrane) and printed material, selecting articles considered by the authors to be of greater scientific and epidemiological relevance. Material published from 1990 to 2007 was consulted.

Discussion: pregnant women have well-defined physiological thyroid changes, such as thyroid enlargement, increased thyroid binding globulin (TBG) in response to increased oestrogen production and transient first trimester hyperthyroidism caused by increased human chorionic gonadotrophin (HCG). These adaptations occur because of maternal interaction with the foetus and placenta; however, women maintain normal thyroid function during normal pregnancies.

Considering such adaptation mechanisms, thyroid function during pregnancy must be evaluated by determining thyrotrophin (TSH) and free thyroxine (free T4); anti-thyroid antibody detection is also useful in some cases.

High doses must be used in substitutive therapy to maintain free T4 level at the normal upper limit from the time diagnosis is established.

Patients having a previous diagnosis of hypothyroidism will usually need dose adjustment, generally increasing levothyroxine by $25 \%$ to $50 \%$.

Patients having positive auto-antibodies during first trimester, especially antiperoxidase auto-antibodies (ATPO) have more risk of transient or permanent post-partum thyroid disease and greater psychiatric symptomatology prevalence. Puerperal follow-up is recommended in such patients.

Key words: hypothyroidism, thyroid, pregnancy.

\section{INTRODUCCIÓN}

Las alteraciones en la función tiroidea son más frecuentes en la mujer; algunos consideran una relación 10:1 respecto de los hombres. ${ }^{1}$ Dentro de ellas, el hipotiroidismo se presenta con una frecuencia del $0,5-2,5 \%$ de todos los embarazos, ${ }^{2}$ mientras que el hipotiroidismo subclínico tiene una prevalencia del 2-5\% en las mujeres embarazadas. Entre el $5-9 \%$ de las pacientes desarrolla enfermedad tiroidea posparto. El hipotiroidismo clínico ha sido asociado con complicaciones gestacionales como partos pretérmino, bajo peso al nacer, abruptio placentae, hipertensión y muerte fetal. ${ }^{3}$ Las hormonas tiroideas maternas desempeñan un rol crucial en el desarrollo del sistema nervioso central del feto, particularmente durante el primer trimestre, debido a la imposibilidad de la tiroides fetal de secretar iodotironinas antes de la semana 10 de gestación. ${ }^{4}$ Hoy en día se acepta que, aun la hipotiroxinemia materna discreta en edades gestacionales tempranas, puede llegar a comprometer el desarrollo psiconeurológico fetal. ${ }^{5}$

En la actualidad se recomienda una ingesta diaria de $200 \mu \mathrm{g}$ de iodo, aunque ciertas poblaciones no logran alcanzar este valor. ${ }^{6}$ Como la tiroxina materna es crucial para la maduración del sistema nervioso fetal, en especial durante el primer trimestre, incluso una discreta deficiencia en la ingesta de iodo podría llegar a ser deletérea. Por lo tanto, el objetivo del presente trabajo es revisar algunos aspectos de la fisiología tiroidea y del hipotiroidismo durante la gestación, como así también su tratamiento y pronóstico con la finalidad de facilitar su manejo en el embarazo por el médico general y el especialista.

\section{Modificaciones fisiológicas en el embarazo}

Durante el embarazo se producen cambios bien definidos en la fisiología tiroidea. Uno de esos cambios es el incremento en los niveles de globulina transportadora de tiroxina (TBG) paralelo al aumento en las concentraciones de estradiol. Esto da como resultado un incremento significativo en el pool de tiroxina total (T4 total), particularmente durante el primer trimestre. ${ }^{7}$ A su vez, la homología estructural entre la gonadotrofina coriónica (hCG) y la tirotrofina (TSH) produce un estímulo sobre sus receptores tiroideos ocasionando estimulación glandular, lo que lleva a un discreto aumento de la 
tiroxina libre (T4L) y una reducción en los niveles de TSH entre la semana 9 y 12 de gestación. ${ }^{8}$ En aproximadamente el $2 \%$ de todos los embarazos, la elevación de la T4L alcanza valores superiores a los normales, que cuando se prolongan, pueden provocar un hipertiroidismo gestacional transitorio, con síntomas y signos más o menos pronunciados de tirotoxicosis. Esta condición se asocia con frecuencia a hiperemesis gravídica. ${ }^{9,10}$ Aproximadamente dos tercios de la tiroxina circulante son transportados por la TBG y esa proporción se incrementa a un 75\% durante el embarazo. El número incrementado de sitios de unión para la tiroxina podrían resultar en una marcada caída en las concentraciones de T4L durante la gestación. Sin embargo, existe un reajuste en el equilibrio de la fracción-libre fracción-unida a la TBG, dada por un aumento en la secreción de hormona por parte de la tiroides. Es posible que el incremento en la concentración de gonadotrofina coriónica pueda jugar un rol en esta homeostasis. En las mujeres embarazadas hipotiroideas, por el contrario, la tiroides no podría responder a dicha estimulación de un modo adecuado, de manera que no existiría un mecanismo de compensación apropiado para el incremento en la disponibilidad de sitios de unión a la TBG. ${ }^{11}$

\section{iSe debe efectuar un tamizaje para hipotiroidismo en las pacientes en edad reproductiva?}

Aún es tema de controversia la pertinencia de efectuar un tamizaje en pacientes en edad reproductiva con deseos de embarazo, a través de la medición de los niveles de TSH y/o tiroxina, con el objeto de detectar una falla tiroidea no reconocida. Algunos autores opinan que si bien no se justificaría un tamizaje "universal”, por lo menos parecería razonable evaluar aquellas mujeres menores de 35 años, con una o más enfermedades autoinmunes órgano-específicas reconocidas, como diabetes (DBT) mellitus tipo 1, o con historia familiar de enfermedad tiroidea. ${ }^{11}$ Vaidya y cols. en un estudio efectuado en 1.560 embarazadas encontraron que un 6,2\% de mujeres presentaban concentraciones anormales de TSH durante la gestación temprana, sin tener factores de riesgo para disfunción tiroidea, y por lo tanto, no hubieran sido reconocidas si solamente se hubiera efectuado un tamizaje para pacientes con factores de riesgo. ${ }^{12}$

Debe tenerse presente que, ofrecer un tamizaje para hipotiroidismo durante la gestación, implica ofrecer eventualmente su tratamiento con levotiroxina, poniendo también en consideración los casos de hipotiroidismo subclínico.

Se han planteado las posibles ventajas y desventajas del tamizaje. Una de las desventajas radicaría en la precocidad con la que debería efectuarse el estudio. Considerando que el desarrollo del cerebro fetal es dependiente de la T4 materna desde la concepción, podría ocurrir que al momento de la primera consulta prenatal el daño ya estuviera establecido. También deben considerarse los costos generados por la realización de la prueba. Con respecto a las ventajas, la elevada prevalencia de la patología tiroidea en el embarazo así como la alta especificidad de la T4L y la TSH plasmática para detectar hipotiroidismo, derivarían en beneficios si se instaurase precozmente una terapia con levotiroxina. $^{13}$

Con relación al rango de referencia de TSH, se ha discutido la conveniencia de redefinir el límite superior normal, fijándolo en 2,5 mU/L, (en lugar del límite habitual de $4 \mathrm{mU} / \mathrm{L}$ ), debido a que aproximadamente el 95\% de la población eutiroidea presenta valores de TSH entre 0,4-2,5 mU/L. Aunque el límite de 2,5 mU/L es el que recomienda actualmente la National Academy of Clinical Biochemistry, ${ }^{14}$ algunos autores consideran que, al menos a la luz de los conocimientos actuales, no existe una evidencia firme que lo sustente. ${ }^{15}$

\section{Hipotiroidismo subclínico y embarazo}

El hipotiroidismo subclínico tiene una prevalencia del 2\% al 5\% en las mujeres embarazadas. ${ }^{2,16}$ Aunque las consecuencias del hipotiroidismo durante 
el embarazo han sido ampliamente estudiadas, no ocurre lo mismo con el hipotiroidismo subclínico, contándose en la actualidad con escasas investigaciones. Casey y cols., en un estudio realizado en el Parkland Hospital de Dallas (Texas), efectuaron comparaciones entre pacientes eutiroideas y con hipotiroidismo subclínico, hallando diferencias: las pacientes con hipotiroidismo subclínico presentaron un riesgo tres veces mayor de abruptio comparado con la población sana, casi el doble de riesgo de parto pretérmino, y mayor dificultad respiratoria y admisión neonatal a cuidados intensivos. No encontraron diferencias en la incidencia de hipertensión gestacional y preeclampsia severa. Sin embargo, los autores no recomiendan el tamizaje sistemático para hallar hipotiroidismo subclínico hasta no poseer la evidencia de mayor cantidad de estudios aleatorizados y controlados. ${ }^{17}$

\section{Hipotiroidismo en el puerperio}

La enfermedad tiroidea posparto se desarrolla entre el 5 y $9 \%$ de las pacientes y se encuentra asociada con la presencia de autoinmunidad tiroidea positiva, especialmente en el primer trimestre de la gestación, adquiriendo relevancia en este periodo la positividad del anticuerpo antiperoxidasa (ATPO). Informes recientes sugieren que puede existir compromiso del coeficiente intelectual en niños nacidos de madres con TSH alta o ATPO detectables durante el embarazo. ${ }^{18}$ Esta enfermedad tiroidea se desarrolla entre las semanas 13 a 19 posparto. Se ha hallado que el 50\% de las pacientes con T4L y TSH normales durante el embarazo, pero con ATPO positivo durante el primer trimestre, desarrolla enfermedad tiroidea posparto, mientras que el otro 50\% permanece eutiroidea, aunque persista el ATPO. $\mathrm{Si}$ bien un gran porcentaje de casos evidencia una disfunción transitoria, aproximadamente el 20-30\% desarrolla hipotiroidismo permanente. A su vez, el seguimiento a largo plazo de las pacientes con disfunción transitoria muestra que un 50\% tiene una recaída a los siete años, esto es, el establecimiento definitivo de un estado hipotiroideo. ${ }^{19}$
Algunos estudios han hallado una incidencia elevada de sintomatología psiquiátrica en las mujeres con ATPO positivo. ${ }^{20,21}$

\section{Evaluación del hipotiroidismo durante el embarazo}

Para estudiar la función tiroidea podemos efectuar determinaciones de tirotrofina, triiodotironina total y libre (T3 y T3L), T4 y T4L. Además, podemos evaluar la positividad de los anticuerpos antitiroideos en sus fracciones antiperoxidasa (ATPO), antitiroglobulina (ATG) y antirreceptor de TSH (TBII, TRab, TSab), que se encuentran generalmente asociados a procesos autoinmunes. Pero, teniendo en consideración las modificaciones fisiológicas propias del embarazo, solo parte de esta batería de estudios será de utilidad.

La cuantificación de T4L permite evaluar la función tiroidea en forma independiente de las variaciones de la TBG y se comporta como un buen indicador de su biodisponibilidad en los tejidos periféricos. ${ }^{22}$ Para el monitoreo clínico de las pacientes hipotiroideas se utilizan tanto la determinación de TSH como la de T4L. El rango de TSH aceptable oscilaría entre 0,5-2,5 mU/L. Se recomienda efectuar nueva determinación de TSH al cabo de 6 a 8 semanas de iniciada la terapia sustitutiva o al modificar una dosis previa de levotiroxina, para dar tiempo a la estabilización del eje tiroideo, logrando un valor más confiable de TSH. ${ }^{23}$ Es recomendable durante el embarazo el estudio de la función tiroidea por lo menos una vez por trimestre.

Con relación a los anticuerpos antitiroideos, los ATPO se hallan presentes con mayor frecuencia. Son citotóxicos y producen diferentes grados de hipotiroidismo. Se considera que a mayor concentración de ATPO, mayor es el grado de evolución de la enfermedad. Como se ha mencionado anteriormente, la presencia de ATPO durante el embarazo se asocia con mayor frecuencia de tiroiditis posparto y sintomatología psiquiátrica puerperal. La utilidad de la determinación de los anticuerpos antitiroglobulina es controvertida, pero se recomienda su cuantifica- 
ción en áreas de bocio endémico y como marcador del cáncer de tiroides. ${ }^{24} \mathrm{El}$ anticuerpo antirreceptor de TSH (TRAb) puede ser estimulante o bloqueante, ocasionando tanto hiper como hipotiroidismo. Es un anticuerpo capaz de atravesar la placenta por lo que puede ocasionar efectos fetales. ${ }^{25}$

\section{Manejo terapéutico}

Se calcula que entre 1-2\% de las gestantes reciben terapia con levotiroxina para tratar su hipotiroidismo. Diversos estudios epidemiológicos indican que $0,4 \%$ de las mujeres embarazadas poseen concentraciones séricas de TSH superiores a $10 \mathrm{mU} / \mathrm{L}$ entre las semanas 15-18 de gestación. ${ }^{26,27}$ Durante el embarazo los requerimientos de hormonas tiroideas se incrementan. Alexánder y cols. efectuaron seguimiento y ajuste de las dosis de levotiroxina en 19 embarazadas hipotiroideas, con la intención de lograr niveles de TSH pregestacionales. La curva de dosis de levotiroxina requerida mostró un rápido incremento entre las semanas 6 y 16 para luego alcanzar una meseta. A las 10 semanas de gestación la dosis de levotiroxina necesaria se incrementó un 29\% aproximadamente respecto de las dosis habituales ( $\mathrm{p}<0,001)$, y a las 20 semanas el incremento necesario fue de un 48\% (p < 0,001), para luego permanecer estable hasta el final de la gestación. El momento en el cual fue observado un incremento en las concentraciones de tirotrofina varió entre las 4,4 y las 16 semanas de gestación (con una media de 8 semanas). ${ }^{28}$

Para el seguimiento de las gestantes hipotiroideas, las recomendaciones actuales de la National Academy of Clinical Biochemistry ${ }^{14}$ incluyen el control del estado tiroideo con TSH y T4L en cada trimestre del embarazo y el ajuste de la dosis de levotiroxina para lograr mantener una TSH sérica entre 0,5-2,5 mUI/L, con una T4L sérica en el tercio superior del intervalo normal de referencia.

\section{CONCLUSIONES}

Durante la gestación no son infrecuentes los trastornos tiroideos. Los efectos de la carencia hormonal materna, así como la autoinmunidad positiva, tienen consecuencias directas sobre la díada maternofetal. Efectuar un tamizaje para hipotiroidismo en pacientes en edad reproductiva es aún motivo de controversia, siendo para algunos pertinente al menos evaluar a las mujeres con factores de riesgo. La presencia de hormonas maternas es de capital importancia para el desarrollo psiconeurológico fetal. El hipotiroidismo durante la gestación ha sido asociado con partos pretérmino, bajo peso al nacer, abruptio placentae, hipertensión y muerte fetal. Si bien son escasas las investigaciones sobre hipotiroidismo subclínico y embarazo, se ha hallado mayor incidencia de complicaciones en estas pacientes. Para el estudio de la función tiroidea durante el embarazo se deberán solicitar los exámenes apropiados, teniendo en cuenta las modificaciones fisiológicas propias de la gravidez. Para el seguimiento de la embarazada hipotiroidea deberá recordarse que los requerimientos de hormona aumentan durante la gestación. El diagnóstico y la terapéutica precoz, redundarán en beneficios tanto para la madre como para el hijo.

\section{REFERENCIAS}

1. Jaksić J, Dumić M, Filipović B, Ille J, Cvijetić M, Gjurić G. Thyroid diseases in a school population with thyromegalia. Arch Dis Child 1994;70:103-6.

2. Klein RZ, Haddow JE, Faix JD, Brown RS, Hermos RJ, Pulkkinen A, et al. Prevalence of thyroid deficiency in pregnant women. Clin Endocrinol (Oxf) 1991;35:41-6.

3. Glinoer D, Soto MF, Bourdoux P, Lejeune B, Delange F, Lemone M, et al. Pregnancy in patients with mild thyroid abnormalities: maternal and neonatal repercussions. J Clin Endocrinol Metab 1991;73:421-7.

4. Utiger RD. Maternal hypothyroidism and fetal development. N Engl J Med 1999;341:601-2.

5. Morreale de Escobar G, Obregón MJ, Escobar del Rey F. Is neuropsychological development related to maternal hypothyroidism or to maternal hypothyroxinemia? J Clin Endocrinol Metab 2000;85:3975-87.

6. Pearce EN, Bazrafshan HR, HE X, Pino S, Braverman LE. Dietary iodine in pregnant women from the Boston, Massachusetts area. Thryroid 2004;14:327-8. 
7. Burrow GN, Fisher DA, Larsen PR. Maternal and fetal thyroid function. N Engl J Med 1994;331:1072-8.

8. Glinoer D, de Nayer P, Bourdoux P, Lemone M, Robyn C, van Steirteghem A, et al. Regulation of maternal thyroid during pregnancy. J Clin Endocrinol Metab 1990;71:276-87.

9. Goodwin TM, Montoro M, Mestman JH, Pekary AE, Hershman JM. The role of chorionic gonadotropin in transient hyperthyroidism of hyperemesis gravidarum. J Clin Endocrinol Metab 1992;75:1333-7.

10. Hershman JM. Human chorionic gonadotropin and the thyroid: hyperemesis gravidarum and trophoblastic tumors. Thyroid 1999;9:653-7.

11. Toft A. Increased levothyroxine requirements in pregnancy-- why, when and how much? N Engl J Med 2004;351:292-4.

12. Vaidya B, Anthony S, Bilous M, Shelds B, Drury J, Hutchison $\mathrm{S}$, et al. Detection of thyroid dysfunction in early pregnancy: Universal screening or targeted high-risk case finding? J Clin Endocrinol Metab 2007;92:203-7.

13. Lazarus JH, Premawardhana LD. Screening for thyroid disease in pregnancy. J Clin Path 2005;58:449-52.

14. Bergoglio LM, Mestman JH. Guía de Consenso para el Diagnóstico y Seguimiento de la Enfermedad Tiroidea. The National academy of clinical biochemistry 2002. Visitado 2007 Jul 3. Disponible en: http://www.aacc. org/NR/rdonlyres/B5FA5D77-BD75-431D-8A60C5E3FD29C3E6/0/thyroid_guidelines_espanol.pdf

15. Brabant G, Beck-Peccoz P, Jarzab B, Laurberg P, Orgiazzi J, Szabolcs I, et al. Is there a need to redefine the upper normal limit of TSH?. Eur J Endocrinol 2006;154:633-7.

16. Woeber KA. Subclinical thyroid dysfunction. Arch Intern Med 1997;157:1065-8.

17. Casey BM, Dashe JS, Wells CE, McIntire DD, Byrd W, Leveno KJ, et al. Subclinical hypothyroidism and preterm birth. Obstet Gynecol 2005;105:239-45.

18. Pop VJ, de Vries E, van Baar AL, Waelkens JJ, de Rooy HA, Horsten M, et al. Maternal thyroid peroxidase antibodies during pregnancy: a marker of impaired child development? J Clin Endocrinol Metab 1995;80:3561-6.

19. Premawardhana LD, Parkes AB, Ammari F, John R,
Darke C, Adams H, et al. Postpartum thyroiditis and long-term thyroid status: prognostic influence of thyroid peroxidase antibodies and ultrasound echogenicity. J Clin Endocrinol Metab 2000;85:71-5.

20. Pop VJ, de Rooy HA, Vader HL, van der Heide D, van Son M, Komproe IH, et al. Postpartum thyroid dysfunction and depression in an unselected population. N Engl J Med 1991;324:1815-6.

21. Harris B, Othman S, Davies JA, Weppner GJ, Richards CJ, Newcombe RG, et al. Association between postpartum thyroid dysfunction and thyroid antibodies and depression. BMJ 1992;305:152-6.

22. Cortelezzi M. Eje tiroideo en la patología gineco-endocrina. En: Sociedad Argentina de Endocrinología Ginecológica y Reproductiva (SAEGRE). Diagnóstico y Terapéutica en Endocrinología Ginecológica y Reproductiva. Buenos Aires: Ed. Ascune Hnos; 2004. p. 96.

23. Demers LM, Spencer CA. NACB: Laboratory Support for the Diagnosis and Monitoring of thyroid Disease. Thyroid 2003;13:33-44.

24. Spencer CA, Takeuchi M, Kazarosyan M, Wang CC, Guttler RB, Singer PA, et al. Serum thyroglobulin autoantibodies: prevalence, influence on serum thyroglobulin measurement and prognostic significance in patients with differentiated thyroid carcinoma. J Clin Endocrinol Metab 1998;83:1121-7.

25. Radetti G, Persani L, Moroder W, Cortelazzi D, Gentili L, Beck-Peccoz P, et al. Transplacental passage of anti-thyroid autoantibodies in pregnant woman with auto-immune thyroid disease. Prenat Diag 1999;19:468-71.

26. Allan WC, Haddow JE, Palomaki GE, Williams JR, Mitchell ML, Hermos RJ, et al. Maternal Thyroid deficiency and pregnancy complications: implications for population screening. J Med Screen 2000;7:12730.

27. Klein RZ, Sargent JD, Larsen PR, Waisbren SE, Haddow JE, Mitchell ML. Relation of severity of maternal hypothyroidism to cognitive development of offspring. J Med Screen 2001;8:18-20.

28. Alexander EK, Marqusee E, Lawence J, Jarolim P, Fischer GA, Larsen PR. Timing and magnitude of increases in Levothyroxine requirements during pregnancy in women with hypothyroidism. N Engl J Med 2004;351:241-9. 